\title{
Caracterización de Estadios del Desarrollo Embrio-Fetal, Vesícula Coriónica y de Placenta en la Gata Doméstica (Felis catus) Mediante Método Ecográfico
}

\author{
Characterization of Stages of Embrio-Fetal, Chorionic Vesicle and Placenta \\ Development in the Domestic Cat (Felis catus) by Ultrasonography
}

Gabriela Illanes*; Claudia Espinoza* \& Patricia Escárate*

ILLANES, G.; ESPINOZA, C. \& ESCÁRATE, P. Caracterización de estadios del desarrollo embrio-fetal, vesícula coriónica y de placenta en la gata doméstica (Felis catus) mediante método ecográfico. Int. J. Morphol., 33(1):178-186, 2015.

RESUMEN: La ecografía es uno de los métodos de diagnóstico gestacional más utilizados, que permite confirmar una gestación temprana y realizar seguimientos del desarrollo embrio-fetal. En este estudio se emplearon 10 gatas gestantes, entre 10 meses a 6 años de edad, con fecha de cruza conocida. Se realizaron exámenes ecográficos seriados en los días 15 (estadio 1), 18 (estadio 2), 21 (estadio 3 ), 38 (estadio 4), y 45 (estadio 5) de gestación para medir parámetros biométricos. Vesícula coriónica: diámetro transversal (DTVC), diámetro longitudinal (DLVC); Placenta: grosor (GP); Feto: longitud céfalo-caudal (LCC), diámetro toraco-abdominal (DTA), diámetro cefálico (DC), tomándose un registro fotográfico de cada estadio. En el estadio 1, no se logró visualizar el embrión. En el estadio 2, el embrión se observó cercano a la pared midiendo en promedio 4,7 milímetros de LCC, el tubo endocárdico se observó funcional. En el estadio 3, se observó el cordón umbilical, pero resultó difícil distinguir los diferentes órganos, excepto el corazón, debido al movimiento causado por sus latidos. En el estadio 4, el feto adquiere movimientos, se observó órganos como el encéfalo, pulmones, el tabicamiento del corazón, diafragma, hígado, estómago, asas intestinales, vejiga y la osificación de huesos. Se observó la flexión de los miembros. En los miembros anteriores se visualizaron los dedos separados, con garras y cojinetes plantares. En el estadio 5, además de las características observadas en el estadio anterior, los riñones muestran la corteza y médula, las cámaras del corazón se visualizaron claramente. Se realizó una correlación lineal entre los parámetros biométricos estudiados y los resultados dieron valor significativo ( $<<0,0001)$ para la mayoría de éstos. Se logró caracterizar los estadios gestacionales estudiados. Siete de las diez gatas presentaron una gestación normal, mientras que una de ellas presentó en uno de sus fetos una malformación congénita y en las otras dos gatas se presentó muerte embrio-fetal.

Palabras clave: Gata; Felis catus; Ecografía; Desarrollo Gestacional

\section{INTRODUCCIÓN}

La ecografía es una técnica de diagnóstico por imagen no invasiva (Moreno et al., 2011) y segura tanto para el profesional como para los pacientes (England \& Harvey, 1998). No requiere una preparación excesiva y tampoco requiere de anestesia o sedación del animal (Feldman \& Nelson 2004). Se utiliza para estudiar tejidos blandos, permitiendo la valorización del tamaño, forma, situación y estructura de los mismos. La ecografía o ultrasonografía se basa en los ultrasonidos generados por un dispositivo llamado transductor, compuesto por cristales piezoeléctricos (Moreno et al.). Generalmente se utilizan cristales artificiales de zirconato-titanato de plomo (PZT), debido a que son muy precisos. Estos cristales experimentan el efecto piezoeléctrico, que consiste en la aplicación de una corriente de alto voltaje en la cara posterior de éstos, provocando que se deformen y logren una vibración. La magnitud de esta deformación y de la frecuencia de vibración es proporcional al voltaje aplicado y se traduce como la fuerza de la onda ultrasónica. No se aplica el voltaje a todos los cristales al mismo tiempo, sino que por fragmentos lineales, para mejorar la calidad de onda. La recepción de estas ondas se logra cuando retornan de nuevo a los cristales después de chocar con los tejidos (Giraldo, 2003).

El ultrasonido son ondas de sonido de alta frecuencia las cuales no son audibles por el hombre. Los sonidos audibles están entre 20-20.000 herzios (Hz ó ciclos por 
segundo) (Giraldo). Dentro de los transductores o sondas que se utilizan en Medicina Veterinaria se encuentran de 3,5, 5 y 7,5 MHz. Para el examen de cuerpos lúteos, folículos y embriones, es conveniente utilizar sondas de 7,5 MHz, ya que la profundidad de penetración es aproximadamente de 5 centímetros (Giraldo). Cuanto mayor es la frecuencia, menor es la profundidad de penetración, pero mayor es la resolución de la imagen (Moreno et al.).

El ecógrafo empleado en Medicina Veterinaria es en modo B (bidimensional) (Giraldo) en donde los ultrasonidos reflejados son representados en el monitor como puntos de diferente brillo (Fritsch \& Gerwing, 1996); y a tiempo real, ya que el movimiento de las estructuras ayuda a la identificación del tejido y de esta manera se podrá estudiar tanto la estructura como su función (Giraldo). También existen otros modos, como el modo M (movimiento), en el cual se utiliza solo un haz de ultrasonidos, los ecos producidos son representados en el monitor de forma continua a lo largo del tiempo, avanzando la imagen hacia la derecha. Se utiliza fundamentalmente en ecocardiografía. En el modo A (amplitud), los ecos reflejados son representados en un oscilógrafo en forma de "picos" de diferentes alturas en función del tiempo (Fritsch \& Gerwing).

El útero en pequeños animales es el órgano más sencillo y rápido de identificar mediante ecografía cuando está alterado. La exploración se realiza con sondas de 7,5 $\mathrm{MHz}$. Se recomienda utilizar sondas lineales en vez de las sectoriales, aunque las primeras pueden dificultar la visualización del cuerpo uterino. La vejiga pletórica sirve como punto de orientación y ventana acústica para localizar y estudiar el cuerpo uterino (Fritsch \& Gerwing).

La gestación en la gata se puede detectar mediante la ecografía a los 10 días (Barr \& Gaschen), 11-14 días después de la cruza (Fritsch \& Gerwing; de Godoy et al., 2010). El diámetro de los sacos gestacionales varía incluso entre los fetos de la misma camada (Dennis et al., 2010). Para evitar diagnósticos de gestación erróneos, se debe diferenciar las vesículas coriónicas de otras estructuras como vasos sanguíneos e intestino delgado, que se encuentran cercanos a la vejiga. Los cortes transversales a través de los vasos sanguíneos que se encuentran adyacentes al útero, pueden tener una gran similitud con las vesículas coriónicas de estadios tempranos de gestación; por esta razón, cuando se duda de la estructura que se visualiza, entonces se debe rotar el transductor, que podrá revelar la forma longitudinal de un vaso sanguíneo o la forma esférica de una vesícula gestacional; además, el pulso de un vaso sanguíneo y los movimientos peristálticos del intestino también ayudarán a su diferenciación (Kähn, 2004).

\section{MATERIAL Y MÉTODO}

Se emplearon 10 gatas gestantes con fecha de cruza conocida, entre 10 meses a 6 años de edad, clínicamente sanas y que presentaron entre 2 a 6 vesículas coriónicas. Las gatas fueron obtenidas de 3 criaderos de la Región Metropolitana ( 5 gatas persas del criadero Gatykey, 2 gatas thai del criadero Cuatro Estaciones, 3 gatas persas del criadero Cuccioli di Facchinetti). A los propietarios se les solicitó firmar un consentimiento informado. Posterior a esto se procedió a la realización de la exploración ecográfica abdominal (Ecógrafo MINDRAY DP 10 año 2012 con transductor microconvexo multifrecuencia 5,0-8,0 Mhz).

La vejiga de cada gata debió estar pletórica para facilitar la visualización de los cuernos uterinos, la paciente se encontraba en ayunas, para evitar que los gases y heces produjeran reverberaciones en las imágenes. Se colocó a la paciente en decúbito dorsal, se aplicó alcohol $95^{\circ}$ sobre el abdomen y gel ecográfico para un mejor acople entre el transductor y la piel de la paciente. Se posicionó el transductor cranealmente al pubis y se exploró ambos cuernos uterinos, con una frecuencia de $8.0 \mathrm{MHz}$. Se realizaron cinco ecografías seriadas, las que se calendarizaron al día $15,18,21,38$ y 45 de gestación a partir de la fecha de la primera cruza. En cada ecografía se midieron los parámetros biométricos: Vesícula coriónica: diámetro transversal (DTVC), diámetro longitudinal (DLVC); Placenta: grosor (GP); Feto: longitud céfalo-caudal (LCC), diámetro toraco abdominal (DTA), diámetro cefálico (DC). En cada estadio se tomó un registro fotográfico (Cámara fotográfica NIKON COOLPIX S630 12 megapixels). Se esperó a que la gestación de cada gata finalizara y se solicitó a sus dueños el número de crías al parto. Los datos fueron analizados con el programa Microsoft Office Excel 2007, en el cual se calculó el promedio, desviaciones estándar, coeficiente de correlación de Pearson r y valor de p entre las distintas variables consideradas.

\section{RESULTADOS}

En la Tabla I se identificó a las hembras felinas durante la gestación, edad, número de la camada, el número de vesículas coriónicas que se observaron mediante el estudio ecográfico y el número de crías al parto.

\section{Caracterización ultrasonográfica del desarrollo gestacional de la gata doméstica.}

Estadío 1 (15 Días). La imagen ecográfica nos muestra dos 
Tabla I. Identificación de las hembras felinas durante el desarrollo gestacional.

\begin{tabular}{|c|c|c|c|c|c|c|c|c|c|}
\hline \multirow{2}{*}{ Gatas } & \multirow{2}{*}{ Edad } & \multirow{2}{*}{$\begin{array}{c}\text { n de la } \\
\text { camada }\end{array}$} & \multicolumn{5}{|c|}{ n de vesículas al día } & \multirow{2}{*}{$\begin{array}{c}\text { Problemas durante la } \\
\text { gestación }\end{array}$} & \multirow{2}{*}{$\begin{array}{c}\text { n de crías a } \\
\text { parto }\end{array}$} \\
\hline & & & 15 & 18 & 21 & 38 & 45 & & \\
\hline Samira & 2 años 8 meses & $4 t^{\mathrm{a}} \mathrm{c}_{\mathrm{amada}}$ & 3 & 3 & 3 & 3 & 3 & no hubo & 4 \\
\hline Lumi & 10 meses & Primeriza & 3 & 3 & 3 & 3 & 3 & no hubo & 5 \\
\hline Zuri & 2 años 9 meses & $3 \mathrm{r}^{\mathrm{a}} \mathrm{c}_{\mathrm{amada}}$ & 4 & 4 & 4 & 4 & 4 & no hubo & 4 \\
\hline Menta & 1 año 3 meses & Primeriza & 2 & 4 & 4 & 5 & 5 & no hubo & 5 \\
\hline Celeste & 2 años 5 meses & $3 \mathrm{r}^{\mathrm{a}} \mathrm{c}_{\mathrm{amada}}$ & 4 & 4 & 4 & 4 & 4 & no hubo & 5 \\
\hline Morocha & 1 año & Primeriza & 2 & 3 & 3 & 3 & 3 & $\mathrm{si}^{(*)}$ & 4 \\
\hline Dolce vita & 1 año 7 meses & Primeriza & 3 & 3 & 3 & 3 & 3 & no hubo & 3 \\
\hline Mili & 1 año & Primeriza & 1 & 2 & 3 & 3 & 3 & $\mathrm{si}^{(* *)}$ & 3 \\
\hline Kiara & 4 años & $4 t^{a} c_{a m a d a}$ & 4 & 4 & 4 & 4 & 4 & $\mathrm{si}^{(* *)}$ & 3 \\
\hline Leonora & 10 meses & Primeriza & 2 & 2 & 2 & 2 & 2 & no hubo & 2 \\
\hline Total & & & 28 & 32 & 33 & 34 & 34 & & 38 \\
\hline
\end{tabular}

$(*)=$ Malformación fetal; $(* *)=$ Muerte embrio-fetal.

zonas anecogénicas. La estructura de la izquierda corresponde a la vejiga (flecha verde), mientras que la de menor tamaño corresponde al lumen de la vesícula coriónica (flecha roja), rodeando a esta zona, a modo de anillo con mayor ecogenicidad se observa el corion y rodeando externamente a éste último se observa el cuerno uterino con menor ecogenicidad (Fig. 1A). Se visualizan dos vesículas coriónicas (flecha roja) (Fig. 1B).

Estadío 2 (18 Días). La zona anecogénica de mayor tamaño corresponde a la vejiga (flecha verde), mientras que la de menor tamaño de forma circular corresponde al lumen de la vesícula coriónica (flecha roja), dentro de la cual se observa adherido a la pared de ésta, una estructura isoecogénica que representa al embrión en desarrollo (flecha amarilla) (Fig. 2A).

Se observa el cordón umbilical isoecogénico (flecha azul) representado por una línea a la izquierda que une el embrión (flecha amarilla) con la pared de la vesícula coriónica (flecha roja) (Fig. 2B).

Estadío 3 (21 Días). En la zona anecogénica que corresponde al lumen de la vesícula coriónica (flecha roja), dentro de ésta, se observa el embrión isoecogénico (flecha amarilla). Con mayor ecogenicidad y de mayor grosor se observa la placenta (flecha naranja), que se encuentra rodeando al lumen de la vesícula coriónica (Fig. 3A). Se observa el cordón umbilical isoecogénico (flecha azul) (Fig. 3B).

Estadío 4 (38 Días). En un plano longitudinal se observa con claridad el ancho de la placenta hipoecogénica (línea naranja) con algunas zonas anecogénicas que corresponde al lumen de la vesícula coriónica (flecha roja). La placenta forma un cinturón que rodea a la vesícula coriónica, dejando los polos anecogénicos craneal y caudal al descubierto (Fig. 4A).

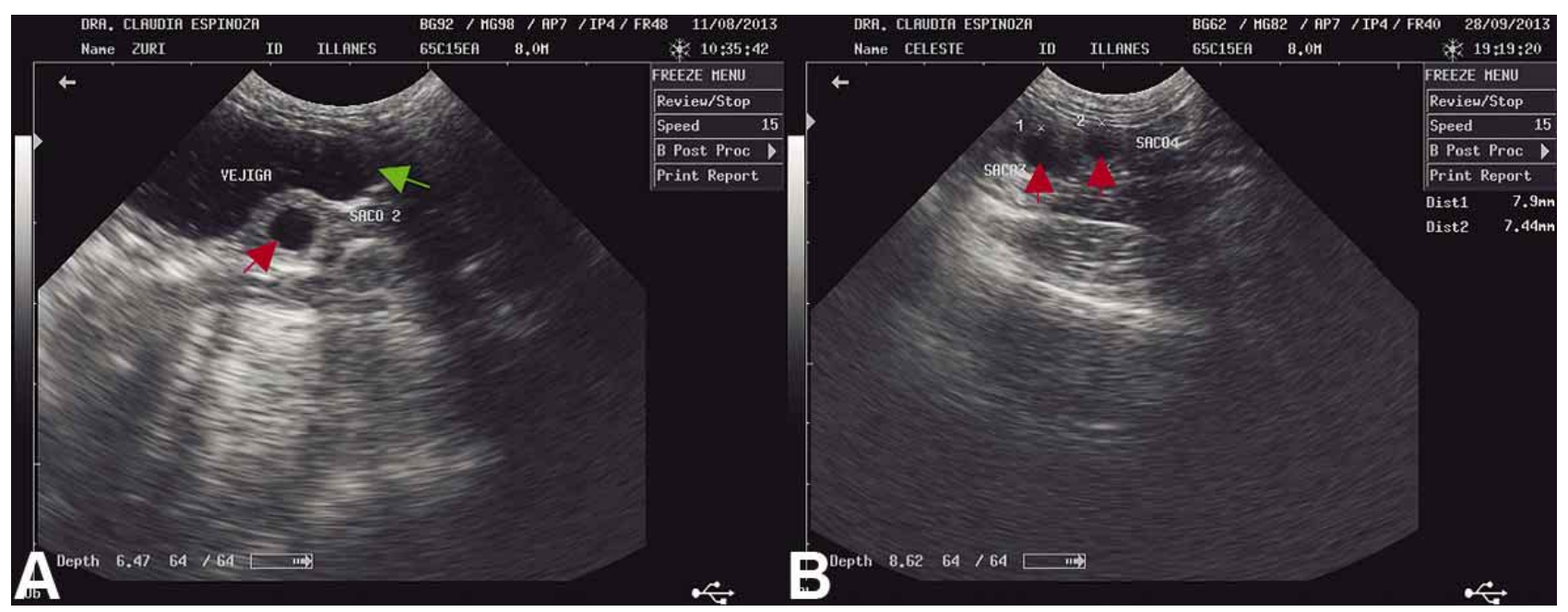

Fig. 1. Muestran corte longitudinal y transversal de la vesícula coriónica o saco gestacional, correspondiente al estadio 1. 


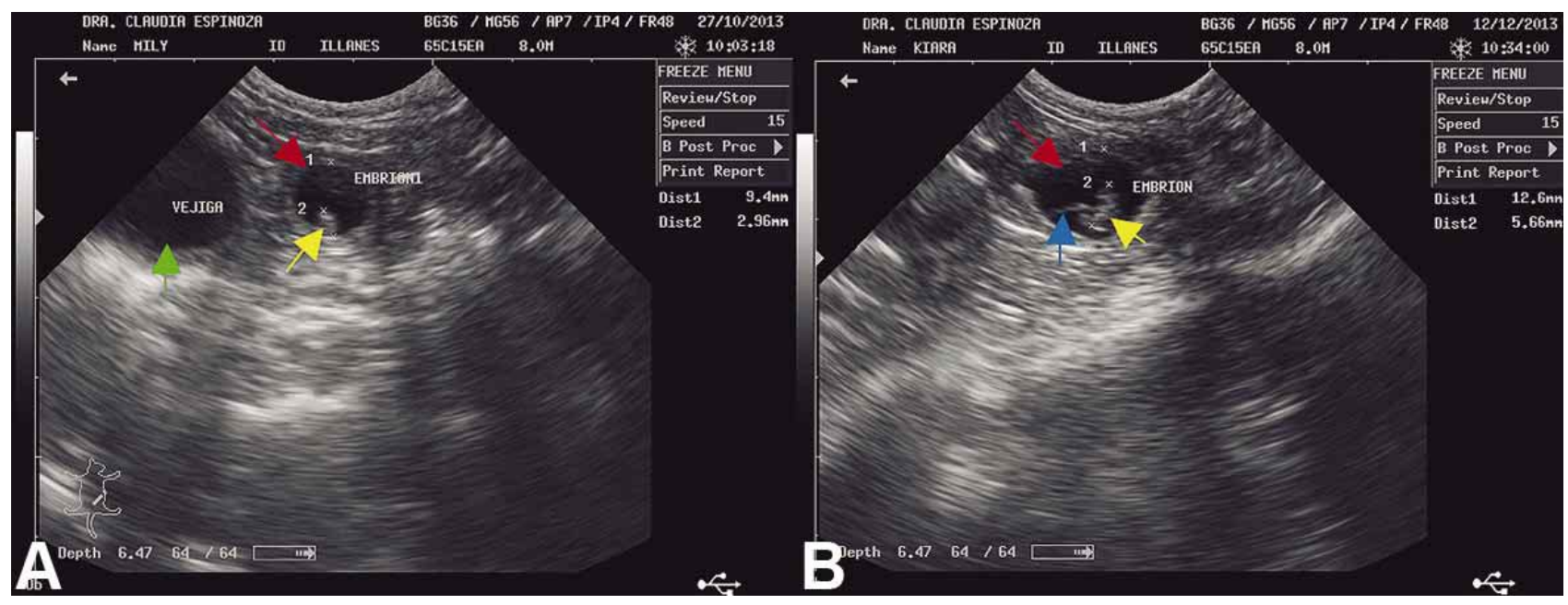

Fig. 2. Muestran corte longitudinal y transversal de la vesícula coriónica o saco gestacional, correspondiente al estadio 2.

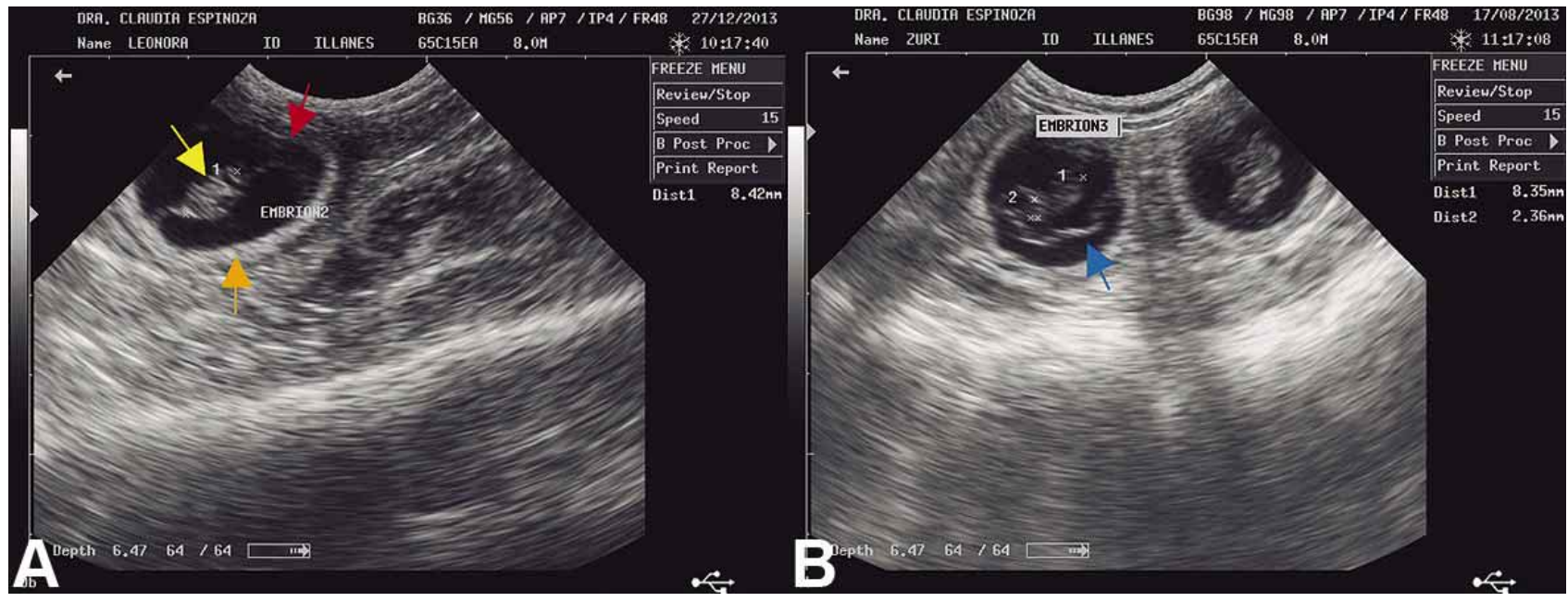

Fig. 3. Muestran corte longitudinal y transversal de la vesícula coriónica o saco gestacional, correspondiente al estadio 3.

Se destaca muy bien el grosor de la placenta (línea naranja), la cual es epiteliocorial (el corion está adherido a la pared del endometrio) formando el cinturón ecuatorial, ésta se observa hipoecogénica con márgenes de mayor ecogenicidad. El lumen de la vesícula coriónica se observa muy reducido, debido al crecimiento fetal que ocupa su mayor parte. Al observar al feto de cefálico a caudal se distingue el cráneo (flecha negra) (en donde la osificación de los huesos de la cabeza se visualiza hiperecogénicos), cuello hipoecogénico, miembros anteriores hipoecogénicos con algunas zonas hiperecogénicas que representan su osificación.

En la región del tórax (línea verde), se observan las costillas hiperecogénicas, corazón hipoecogénico y pulmones con mayor

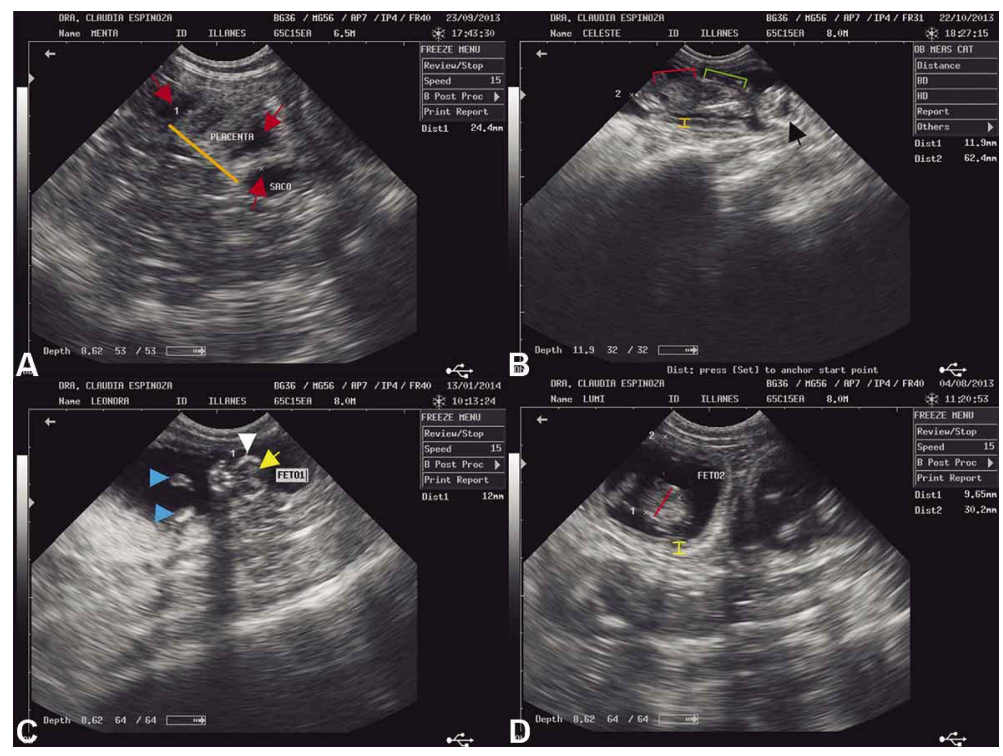

Fig. 4. Muestran corte longitudinal y transversal de la vesícula coriónica o saco gestacional, correspondiente al estadio 4 . 
ecogenicidad, el diafragma se distingue porque se observa entre los diferentes patrones de ecogenicidad de pulmones e hígado. En la región abdominal (línea roja) se visualiza hígado con menor ecogenicidad, asas intestinales con mayor ecogenicidad y vejiga anecogénica. Los miembros posteriores se observan hipoecogénicos con zonas hiperecogénicas que muestran el proceso de osificación. En este estadio de observaron movimientos fetales (Fig. 4B).

Se observa la cabeza del feto, destacando con patrón hiperecogénico los huesos parietales y nasales (flecha blanca). El encéfalo (flecha amarilla) se visualiza hipoecogénico, las cuencas de los ojos se observan con poca ecogenicidad. Se observan los miembros anteriores (flecha azul) hipoecogénicos con los extremos (manos) hiperecogénicos. Se distinguen las garras hiperecogénicas y cojinetes plantares con menor intensidad de ecogenicidad (Fig. 4C).

Se observa un corte transversal de la vesícula coriónica, en la que se distingue el diámetro tóraco-abdominal (línea roja). La placenta (línea amarilla) se observa gruesa y nítida con mayor ecogenicidad (Fig. 4D).

Estadío 5 (45 Días). Se observa la cabeza del feto, en la que se destaca más su cara, las cuencas de los ojos (flecha amarilla) con menor ecogenicidad, el encéfalo hipoecogénico (flecha roja), huesos parietales y nasales hiperecogénicos (flecha blanca) (Fig. 5A).

Debido al gran tamaño del feto, sólo se aprecia el tórax y abdomen, en tórax se distingue el corazón (flecha roja) hipoecogénico con algunas zonas anecogénicas representando sus cámaras, éste abarca la mayor parte del tórax, las costillas (flecha verde) se visualizan hiperecogénicas. Separando el tórax del abdomen, se encuentra el diafragma, éste se distingue por la diferencia de ecogenicidad entre pulmones e hígado. En la región abdominal, se observa hígado (flecha blanca) con menor ecogenicidad, estómago (flecha blanca) y vejiga (flecha amarilla) anecogénicos (Fig. 5B).

Se observa la región abdominal, en la que se distingue estómago (E) y vejiga (V) anecogénicos, en el riñón (flecha roja) se distingue la corteza hipoecogénica y la médula con menor ecogenicidad. Se observa parte de la columna (flecha amarilla) hiperecogénicos, miembros posteriores (flecha azul) con zonas de diferentes ecogenicidad. La placenta (flecha verde) se observa gruesa (Fig. 5C).

Se aprecia el tórax y abdomen, en tórax se distingue el corazón (flecha roja) hipoecogénico. Se observan pulmones (flecha azul) con mayor ecogenicidad. El diafragma se puede distinguir a través de la diferencia de ecogenicidad entre pulmones e hígado. En la región abdominal, las asas intestinales (flecha verde) presentan mayor ecogenicidad que el hígado (flecha naranja), estómago anecogénico (flecha blanca) (Fig. 5D).

En la Tabla II se observan los promedios (P) y desviación estándar (DS) de las variables de los estadios considerados en este estudio.

Tabla II. Promedio y Desviación Estándar de las variables del desarrollo gestacional de la gata doméstica.

\begin{tabular}{|c|c|c|c|c|c|c|c|c|c|}
\hline \multirow{2}{*}{ Día } & \multirow{2}{*}{ Estadio } & \multirow{2}{*}{$\begin{array}{c}n \\
\text { Individuos }\end{array}$} & & \multicolumn{3}{|c|}{ Embrión/ Feto } & \multirow{2}{*}{$\begin{array}{c}\text { Placenta } \\
\text { GP }\end{array}$} & \multicolumn{2}{|c|}{ Vesícula Coriónica } \\
\hline & & & & LCC & DTA & DC & & DTVC & DLVC \\
\hline \multirow[t]{2}{*}{15} & 1 & 28 & $\mathbf{P}$ & --- & --- & --- & --- & 5,892 & 7,136 \\
\hline & & & DE & --- & --- & --- & --- & 1,339 & 1,652 \\
\hline \multirow[t]{2}{*}{18} & 2 & 32 & $\mathbf{P}$ & 4,748 & --- & --- & --- & 11,241 & 13,028 \\
\hline & & & DE & 0,899 & --- & --- & --- & 1,811 & 2,462 \\
\hline \multirow[t]{2}{*}{21} & 3 & 33 & $\mathbf{P}$ & 7,596 & --- & 1,588 & 2,071 & 16,158 & 19,061 \\
\hline & & & DE & 1,638 & --- & 0,463 & 0,522 & 1,829 & 2,971 \\
\hline \multirow[t]{2}{*}{38} & 4 & 32 & $\mathbf{P}$ & 48,506 & 10,546 & 11,124 & 4,403 & 31,534 & 63,216 \\
\hline & & & DE & 6,278 & 1,342 & 0,903 & 0,619 & 4,313 & 6,966 \\
\hline \multirow[t]{2}{*}{45} & 5 & 32 & $\mathbf{P}$ & 67,803 & 15,775 & 15,297 & 4,760 & 36,906 & 86,406 \\
\hline & & & DE & 8,613 & 1,821 & 1,292 & 1,271 & 3,658 & 18,807 \\
\hline
\end{tabular}

Tabla III. Coeficiente de correlación de Pearson (r).

\begin{tabular}{lllllll}
\hline & LCC & DTA & DC & GP & DTVC & DLVC \\
\cline { 2 - 7 } LCC & 1,000 & 0,996 & 0,998 & 0,939 & 0,979 & 0,999 \\
DTA & 0,996 & 1,000 & 0,995 & 0,825 & 0,228 & 0,818 \\
DC & 0,998 & 0,995 & 1,000 & 0,949 & 0,978 & 0,998 \\
GP & 0,939 & 0,825 & 0,949 & 1,000 & 0,977 & 0,947 \\
DTVC & 0,979 & 0,228 & 0,978 & 0,977 & 1,000 & 0,984 \\
DLVC & 0,999 & 0,818 & 0,998 & 0,947 & 0,984 & 1,000 \\
\hline
\end{tabular}




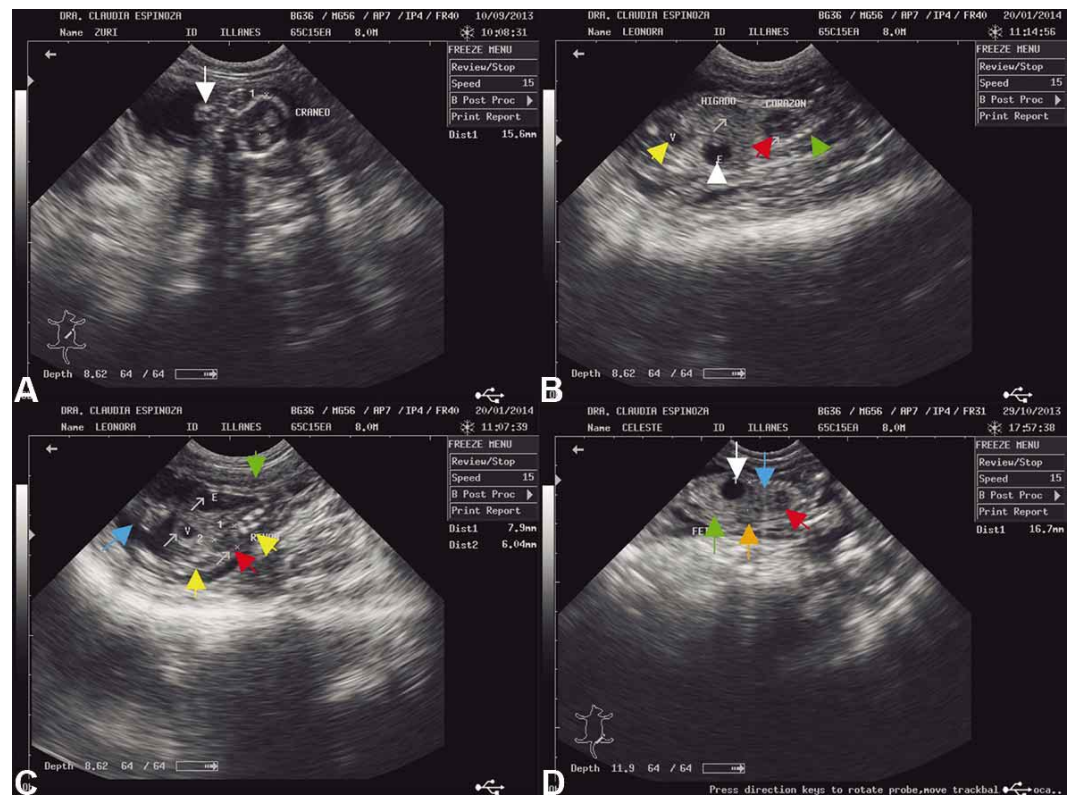

Fig. 5. Muestran corte longitudinal y transversal de la vesícula coriónica o saco gestacional, correspondiente al estadio 5 .

Tabla IV. Valores de $\mathrm{p}$.

En la Tabla III se describe el cálculo del coeficiente de correlación de Pearson ( $r$ ) entre las diferentes variables, obteniéndose en todas un resultado positivo.

En la Tabla IV se observan los valores de $p$ entre las diferentes variables, siendo los parámetros que dieron un valor altamente significativo DTA vs LCC, DC vs LCC, DC vs DTA, GP vs LCC, GP vs DC, DTVC vs LCC, DTVC vs DC, DTVC vs GP, DLVC vs LCC, DLVC vs DC, DLVC vs GP y DLVC vs DTVC, en los que se muestra una correlación positiva $(\mathrm{p}<0,0001)$. Entre las variables GP vs DTA, DTVC vs DTA, DLVC vs DTA, el valor de p es mayor a 0,0001 y no se muestra una correlación entre ellas. La correlación de parámetros biométricos se observa en la Figura 6.

Fig. 6. Correlación de parámetros biométricos: LCC, DTA, DC, GP, DTVC, DLVC. Eje Y: Unidad de medida en milímetros (mm). Eje X: Estadios ecográficos analizados en el desarrollo gestacional.Vesícula Coriónica: Diámetro Longitudinal (DLVC), Diámetro Transversal (DTVC); Placenta: Grosor (GP); Embrión-Feto: Diámetro Cefálico (DC), Diámetro Toraco Abdominal (DTA), Longitud Céfalo-Caudal (LCC).

\begin{tabular}{lllllll}
\hline & LCC & DTA & DC & GP & DTVC & DLVC \\
\cline { 2 - 7 } LCC & 0,000000 & 0,000008 & 0,000001 & 0,005218 & 0,000388 & 0,000000 \\
DTA & 0,000008 & 0,000000 & 0,000012 & 0,052351 & 0,701775 & 0,056741 \\
DC & 0,000001 & 0,000012 & 0,000000 & 0,003428 & 0,000485 & 0,000002 \\
GP & 0,005218 & 0,052351 & 0,003428 & 0,000000 & 0,000531 & 0,003775 \\
DTVC & 0,000388 & 0,701775 & 0,000485 & 0,000531 & 0,000000 & 0,000208 \\
DLVC & 0,000000 & 0,056741 & 0,000002 & 0,003775 & 0,000208 & 0,000000 \\
\hline
\end{tabular}

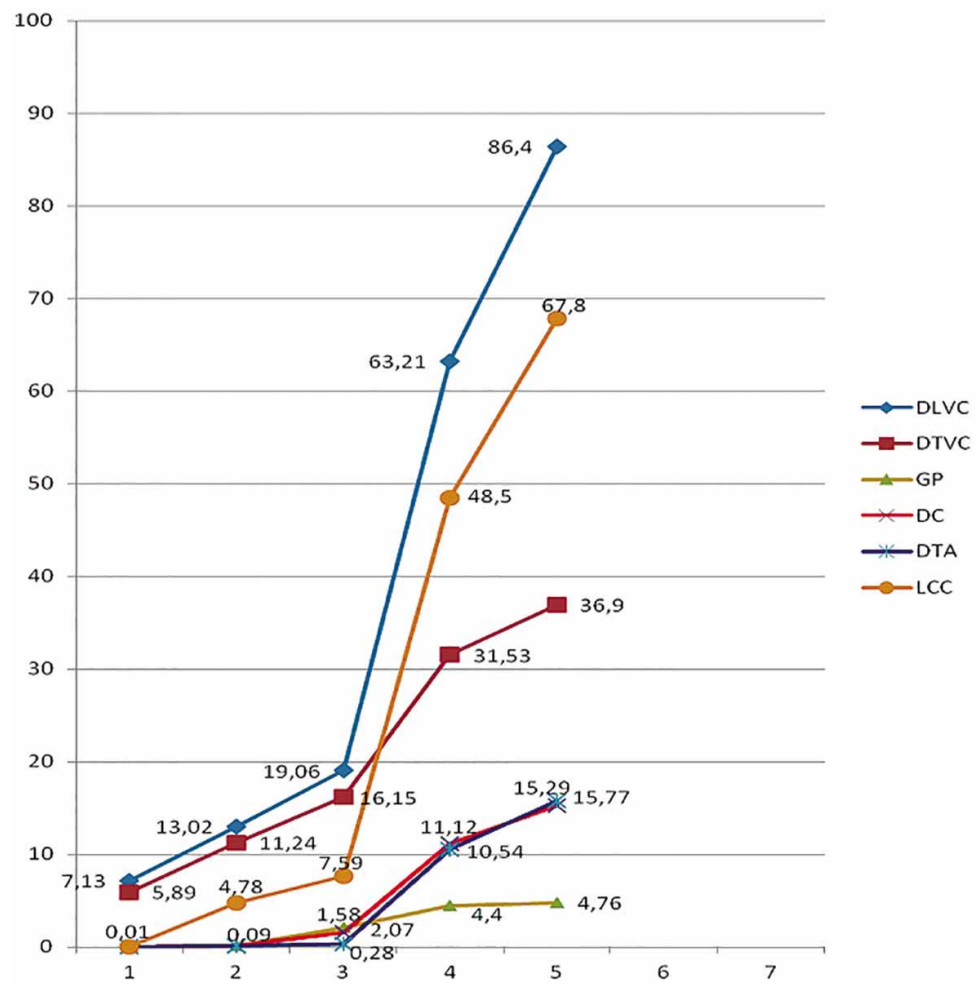




\section{DISCUSIÓN}

Se ha descrito con bastante detalle las estructuras y procesos que ocurren en los diferentes estadios analizados durante el desarrollo gestacional de la hembra felina, Dennis et al., Kähn, Illanes et al. (2007), de Godoy et al., Barr \& Gaschen, entre otros. Sin embargo, éstos no se reconocen fácilmente mediante la ultrasonografía, pero si son muy útiles como referencia para el diagnóstico clínico. Se logró así caracterizar cinco estadios que permiten realizar un diagnóstico gestacional temprano mediante ecografía, asociando lo ya descrito morfológicamente y lo observado con ultrasonografía.

En el estadio 1, correspondiente a los 15 días del desarrollo gestacional, se puede observar las vesículas coriónicas al interior de los cuernos uterinos. Kähn, señala que las primeras vesículas coriónicas no presentan ninguna ecogenicidad embrionaria en su interior. Se puede determinar también que para un mismo estadio, los diámetros de las vesículas coriónicas varían, inclusive entre los fetos de una misma camada, Dennis et al.

Morfológicamente el embrión es trilaminar plano, que no hace prominencia hacia el lumen y se encuentra adherido a la pared del endometrio (epitelio-corial). Por tanto no evidenciable al ultrasonido. Sin embargo presenta un corazón tubular funcional (Knospe, 2002; Illanes et al.).

En el estadio 2 (18 días de desarrollo gestacional), se observa un embrión hipoecogénico cercano a la pared del saco coriónico, al respecto Barr \& Gaschen señalan que el embrión se puede observar a partir de los 16 a 18 días. El latido cardiaco se percibe nítidamente, hecho observado por de Godoy et al., entre los 15 y 17 días de gestación, Nelson \& Couto (2000), entre los 18 y 25 días; Illanes et al., describen histológicamente el tubo endocardico a los 15 días y el tabicamiento cardiaco a los 18 días.

En el estadio 3 (21 días de desarrollo gestacional), las vesículas coriónicas aún presentan forma de esfera, la placenta aumenta de grosor, con mayor ecogenicidad y se vuelve más nítida. El embrión se observa más grande e isoecogénico, se observa el cordón umbilical, el corazón latiendo, sin embargo no se logra diferenciar otros órganos. A los 20 días, las extremidades anteriores y posteriores muestran la flexura del codo y del corvejón, respectivamente (Illanes et al.).

En el estadio 4 (38 días de desarrollo gestacional), Kähn describe que el útero adopta una forma más tubular hacia los días 35-40 de gestación. El diámetro transversal de la vesícula coriónica alcanza entre $25-35 \mathrm{~mm}$ y el diá- metro longitudinal entre 60-80 mm, estas medidas coinciden con las de este estudio. Con respecto al feto, es factible observar movimientos, los que se caracterizan por la flexión de la cabeza y la extensión de los miembros (Nelson \& Couto). Kähn describe que entre los 35-40 días, la organogénesis ha progresado de forma considerable, ya que se puede reconocer el desarrollo de los diferentes órganos, lo que se ratifica con este estudio, ya que se pudo visualizar el encéfalo hipoecogénico, pulmones hipoecogénicos debido a que aún no poseen aire en su interior y corazón con menor ecogenicidad.

El proceso de osificación del cráneo, columna, costillas y miembros, se evidencia por una alta hiperecogenicidad. Según el estudio de Dennis et al., este proceso se detecta entre los 30-33 días de gestación. Mannion (2006) señala que a los 35 días se aprecia la osificación con patrón hiperecogénico y a los 40 días se observa la sombra acústica de forma más extendida en la pantalla del ecógrafo, mostrando así el progreso de calcificación de los huesos. En este estudio, dichas características fueron observadas en el día 38 de gestación.

En la región abdominal se puede distinguir el hígado con menor ecogenicidad produciéndose un contraste con respecto a los pulmones, entre los que se puede distinguir el diafragma, representado por una línea que abarca todo el diámetro tóraco-abdominal. Las asas intestinales se visualizan con mayor ecogenicidad que el hígado. El estómago y la vejiga urinaria se muestran anecogénicos. Esto tiene concordancia con el estudio realizado por Mannion. Dennis et al., mencionan que a los 37 días se distinguen las cámaras del corazón mediante ecografía. En este estadio se visualizan las cámaras cardiacas.

Knospe menciona que a los 32-38 días, el feto crece rápidamente, la cabeza se observa más grande que la región del tronco, el cordón umbilical se visualiza más delgado, los miembros poseen la típica posición fetal, en donde los codos y los corvejones están flexionados. Los dedos de los miembros anteriores y posteriores y la queratinización de las garras están claramente definidos. En este estudio las garras y cojinetes plantares se pueden observar muy claros en la Figura 4C. Knospe describe además, que en la cavidad torácica se encuentra en desarrollo el miocardio, vasos y bronquios. En la región abdominal, el estómago y riñones. Osificación de los huesos parietales, frontales, nasales. Algunas estructuras mencionadas como vasos, bronquios, no son evidenciables mediante ultrasonografía, debido a su tamaño reducido y poca ecogenicidad. 
En el estadio 5 (45 días de desarrollo gestacional), se visualizó en la región abdominal los riñones. de Godoy et $a l$, describen que entre los 44-45 días, los riñones dejan de presentar un patrón anecogénico y se puede diferenciar la corteza de la médula. Además, en este estadio se visualizó con mayor claridad el corazón tetracameral.

Según Mannion, se puede determinar el número de fetos si la ecografía se realiza entre los 27-37 días de gestación. El tamaño de la camada es más fácil de determinar en la gestación temprana y cuando la camada es pequeña. No obstante, la reabsorción embrionaria o fetal puede ocurrir, por lo que sólo se puede realizar una estimación del tamaño de la camada (Barr \& Gaschen). En el presente trabajo, hubo gatas a las cuales se contabilizó menos crías de las que tuvieron en el parto, esto se puede deber a que las heces y gases intestinales impidieron la visualización, además la reabsorción embrionaria-fetal ocurrió en dos gatas del estudio.

La placenta felina es endoteliocorial y zonal (Ettinger \& Feldman, 2010). Según Kähn, en la gestación temprana, la placenta abarca prácticamente toda la vesícula coriónica, la cual forma un cinturón alrededor del feto que se visualiza moderadamente hipoecogénica. Los bordes de la placenta zonal, se enroscan en dirección contraria del endometrio y se proyecta ligeramente hacia el lumen de la vesícula coriónica (zona marginal). La placenta es más gruesa que la pared uterina, lo que concuerda con lo observado en los estadios 4 y 5 de este estudio.

El análisis estadístico de correlación lineal (r) de las variables analizadas (DTA vs LCC, DC vs LCC, DC vs DTA, GP vs LCC, GP vs DC, DTVC vs LCC, DTVC vs DC, DTVC vs GP, DLVC vs LCC, DLVC vs DC, DLVC vs GP y DLVC vs DTVC) en estos estadios presenta un alto grado de significancia $(p<0,0001)$ lo que indica una correlación positiva entre ellas y que entre estas variables dependen unas de las otras durante la gestación, mientras que las variables GP vs DTA, DTVC vs DTA y DLVC vs DTA, el valor de p es mayor a 0,0001, lo cual indica que entre estas variables no se puede refutar el hecho de que exista como no exista una correlación con lo visto en el presente estudio (Tabla III los resultados fueron positivos) por lo que se infiere que estas variables son independientes entre sí, a pesar de que todas van en crecimiento.

AGRADECIMIENTOS. A Paola Yañez (Gatykey Cattery), Daniela Guerra (Cuatro Estaciones Cattery), Piero Facchinetti (Cuccioli di Facchinetti Cattery), Patricia López por su confianza y buena disposición al facilitarnos sus gatas, ya que sin su apoyo no habría sido posible este estudio.
ILLANES, G.; ESPINOZA, C. \& ESCÁRATE, P. Characterization of stages of embrio-fetal, chorionic vesicle and placenta development in the domestic cat (Felis catus) by ultrasonography. Int. J. Morphol., 33(1):178-186, 2015.

SUMMARY: Ultrasound is one of the most widely used methods for gestational diagnosis, allowing the detection of early pregnancy and evaluation of fetal growth and development. Ten pregnant cats (aged 10 months to-6 years) were used in this study, with known dates of mating. Serial ultrasound testings were performed on days 15th (stage 1), 18th (stage 2), 21st (stage 3), 38 th (stage 4 ) and 45th (stage 5) of gestation, to measure biometric parameters (Chorionic vesicle: transverse diameter (DTVC), longitudinal diameter (DLVC); Placenta: thickness (GP); Fetus: cephalocaudal length (LCC), thoraco-abdominal diameter (DTA), cephalic diameter (DC). Photographic records were made at each stage. In stage 1, it was impossible to visualize the embryo. In stage 2 , the embryo was observed near the wall measuring $4.7 \mathrm{~mm}$ on average LCC, and the endocardial tube was functioning. In stage 3 , the umbilical cord was observed, but it was difficult to distinguish different organs, with the exception of the heart, which was detected by heartbeat movements. In stage 4 , the fetus begins to move. Organs, such as the brain, lungs, heart septation, diaphragm, liver, stomach, bowel, bladder and bone ossification were observed. In the forelimbs, fingers apart with claws and footpads were visualized. In stage 5 , in addition to the features observed in the previous stage, the kidneys show the cortex and medulla, and the heart chambers are clearly visualize. A linear correlation analysis among the biometric parameters was performed. Results were statistically significant for most parameters studied $(p<0.0001)$. We characterized the gestational stages studied. Out of the ten cats studied, seven cats had a normal pregnancy. One cat showed one congenitally malformed fetus, and one fetal death occurred in the other two cats.

KEY WORDS: Cat; Felis catus; Ultrasonography; Gestational Development.

\section{REFERENCIAS BIBLIOGRÁFICAS}

Barr, F. J. \& Gaschen, L. BSAVA Manual of Canine and Feline Ultrasonography. London, BSAVA, 2011. Pp.172-4.

de Godoy, C. L. B.; Pellegrini, L. C.; Santarosa, I. M. \& Krolikowski, G. Diagnóstico por imagem em medicina veterinária. Santa Maria, Ed.da Universidade Federal de Santa Maria, 2010.

Dennis, R.; Kirberger, R. M.; Barr, F. \& Wrigley, R. H. Handbook of small animal radiology and ultrasound: techniques and differential diagnoses. 2nd ed. Edinburgh, Elsevier, 2010.

England, G. \& Harvey, M. Manual of Small Animal Reproduction and Neonatology. Quedgeley, BSAVA, 1998. pp.114-8. 
ILLANES, G.; ESPINOZA, C. \& ESCÁRATE, P. Caracterización de estadios del desarrollo embrio-fetal, vesícula coriónica y de placenta en la gata doméstica (Felis catus) mediante método ecográfico. Int. J. Morphol., 33(1):178-186, 2015.

Ettinger, S. J. \& Feldman, E. C. Textbook of Veterinary Internal Medicine: Diseases of the Dog and the Cat. $7^{\mathrm{a}}$ ed. St. Louis, Saunders, 2010. pp.5427-33.

Feldman, E. C. \& Nelson, R. W. Canine and Feline Endocrinology and Reproduction. $3^{\mathrm{a}}$ ed. St. Louis, Saunders, 2004. pp.176689.

Fritsch, R. \& Gerwing, M. Ecografía de Perros y Gatos. Zaragoza, Ed. Acribia, 1996. pp.79-80.

Giraldo E., C. Principios básicos de ultrasonografía veterinaria. MVZ Cordoba, 8(2):303-9, 2003. Disponible en: http:// revistas.unicordoba.edu.co/revistamvz/MVZ-82/303.pdf

Illanes, J.; Orellana, C.; Fertilio, B.; Leyton, V. \& Venegas, F. Macroscopic and Microscopic Analysis of the Embryonic and Fetal Growth in the Cat (Felis catus), in Relation to Chorionic Vesicle and Placental Development. Int. J. Morphol., 25(3):467-81, 2007.

Kähn, W. Veterinary Reproductive Ultrasonography. Hannover, Schlütersche, 2004. pp.227-49.

Knospe, C. Periods and stages of the prenatal development of the domestic cat. Anat. Histol. Embryol., 31(1):37-51, 2002.

Mannion, P. Diagnostic Ultrasound in Small Animal Practice. Iowa, Blackwell Publishing, 2006. pp.148-9.

Moreno, B. A.; Hervás, R. J. \& Chacón, F. Significado patológico de la imagen ultrasónica en pequeños animales. An. R. Acad. Cienc. Vet. Andal. Orient., 24(1), 2011. Disponible en: http:// dialnet.unirioja.es/descarga/articulo/4247460.pdf.

Nelson, R. W. \& Couto, C. G. Medicina Interna de Animales Pequeños. $2^{\mathrm{a}}$ ed. Buenos Aires, Intermedica, 2000. pp.896-939.

\author{
Dirección para Correspondencia: \\ Gabriela Illanes Troncoso \\ Médico Veterinario \\ Universidad Iberoamericana de Ciencias y Tecnología \\ Padre Miguel Olivares No1620 \\ Santiago \\ CHILE
}

Email: gabillanes.vet@gmail.com

Recibido: 08-10-2014

Aceptado: 30-12-2014 